\title{
Gene Interactions Affecting Methionine Biosynthesis and the Response to S-methylcysteine by Mutants of Neurospora crassa
}

\author{
By S. TOKUNO, B. STRAUSS AND Y. TSUDA \\ Department of Microbiology, The University of Chicago, Chicago 37, Illinois, U.S.A.
}

(Received 12 September 1961)

SUMMARY

Two non-allelic suppressors have been isolated which suppress nonallelic, leaky, methionine-requiring mutants blocked either between cysteine and cystathionine or between cystathionine and homocysteine. Strains carrying either suppressor gene in the presence of the $m e^{+}$allele were stimulated by either cysteine or methionine, whereas the suppressed methionine-requiring mutants ( $m e s u$ ) although stimulated by methionine were inhibited by cysteine. S-methylcysteine supported the growth of leaky methionine-requiring mutants when it was present as the sole sulphur source and it also stimulated the growth of suppressed methioninerequiring mutants inhibited by cysteine. Sulphate or cysteine inhibited the growth response of certain methionine-requiring mutants to Smethylcysteine. The incorporation of radioactive sulphate into protein methionine was inhibited to a greater extent by S-methylcysteine than was its incorporation into protein cysteine. The results suggest that the sulphur of S-methylcysteine is converted to methionine without prior conversion to cysteine and that the suppressors act by retarding the formation of an inhibitor which accumulates as a result of the primary mutation to methionine requirement.

\section{INTRODUCTION}

It has been pointed out before (Strauss, 1955) that the phenotype of a double mutant cannot always be simply deduced from the properties of the single mutants. In the case of suppressor mutations, it has been repeatedly observed that single auxotrophic mutants can be induced to grow prototrophically by the introduction of a second mutant gene. Such suppressor genes often show a marked degree of specificity (Yanofsky \& Bonner, 1955); certain suppressors are active only on particular mutant alleles but will not suppress other, closely linked, alleles of the same mutant gene. Consequently, the demonstration by Giles (1951) that a single suppressor could suppress two unlinked genes controlling different (but sequential) steps in the biosynthesis of methionine appeared of interest to us. We therefore investigated the characteristics of this type of suppressor to determine whether a new type of gene interaction was represented. Our results indicate that in methionine biosynthesis, these particular suppressors act by preventing the formation of an inhibitor accumulated as a result of the original mutation to auxotrophy, an action similar in nature to that of the suppressors of the acetate-requiring mutants (Strauss \& Pierog, 1954). In the course of our studies, some observations were 
made on the response of methionine-requiring mutants to S-methylcysteine. These results support the idea of Wiebers \& Garner (1960) and of Maw (1961) that Smethylcysteine can act as a methionine precursor without prior conversion to cysteine.

\section{METHODS}

Organisms. The methionine-requiring mutants used in this study were isolated by the filtration-selection method of Woodward, De Zeeuw \& Srb (1954). Strains M3, M 4 and M17 were isolated from among conidia of wild-type Neurospora crassa strain 74 A after treatment with ultraviolet (u.v.) radiation; strains $M$ 7 and M 14 were isolated from material treated with diethyl sulphate. Although M3 and M4

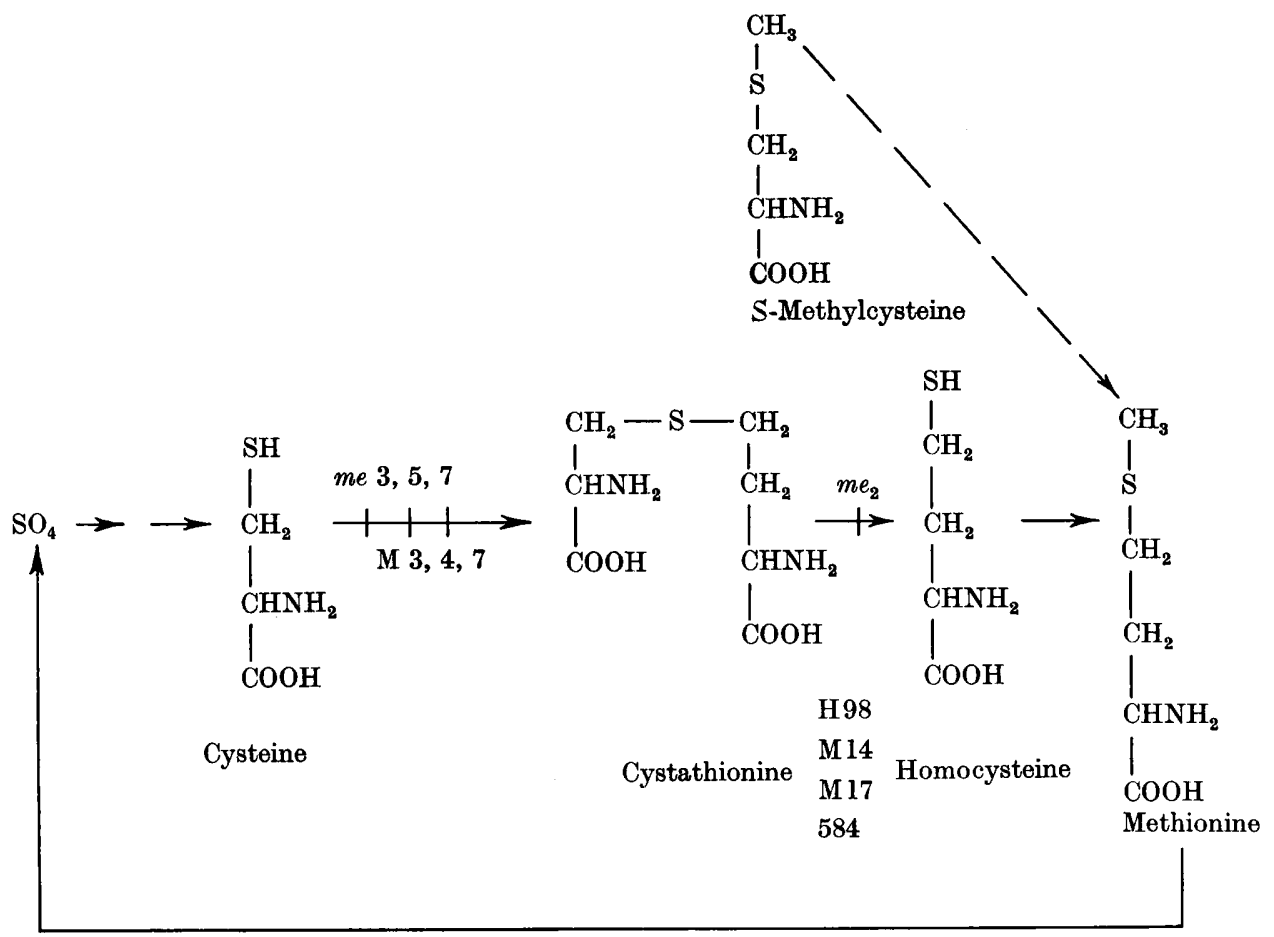

Fig. 1. Scheme of methionine biosynthesis in Neurospora.

and $M 7$ respond to either cystathionine, homocysteine or methionine, but not to cysteine, these strains are not alleles. M3 formed heterocaryons with both M4 and M7, and in crosses of $\mathbf{M 3} \times \mathbf{M} 4$ and of M3 $\times$ M7 a high percentage of wild-type recombinants was recovered. Strains M14 and M17 respond to methionine and homocysteine but not to either cysteine or cystathionine. A simplified pathway of methionine biosynthesis indicating the respective nutritional blocks is shown in Fig. 1.

Strain H98 was re-isolated from a culture sent to us by $\mathrm{Dr}$ N. H. Horowitz. This strain has been used previously in a number of investigations (Giles, 1951; Wiebers \& Garner, 1960) and responds to homocysteine but not to cystathionine in the standard growth test. The suppressor strain $s u^{\text {H98 }}$ was isolated from a culture of H98 which grew on minimal medium. A further suppressor $s u^{\mathbb{M 4}}$ was isolated by 
the method of Yanofsky \& Bonner (1955) from M4 conidia irradiated with u.v. radiation and plated on a minimal medium. Strain 74A of Neurospora crassa was used as a wild-type control. In this paper we use both isolation numbers and also the designation of the allele. Strain $\mathrm{H} 98$ is a mutant at the me-2 locus and is therefore written $m e-2^{\text {म98 }}$. Suppressor genes are identified by the strain from which they originated, i.e. $m e-2^{\mathrm{H} 98} s u^{\mathrm{M} 4}$ represents the double mutant in which $\mathrm{H} 98$ is combined with a suppressor gene originating in strain M4. All strains responding to homocysteine but not to cystathionine are classified as $m e-2$ in this paper, but since three loci have been reported to yield mutants responding to cystathionine but not to cysteine (Barratt, Newmeyer, Perkins \& Garnjobst, 1954), we have merely indicated such strains as me.

Nutritional and biochemical methods. These methods were described previously (Strauss \& Minagawa, 1959). Cystathionine was used as DL allocystathionine, homocysteine as homocysteine thiolactone and S-methylcysteine as the L form. All were commercial preparations and were autoclaved in the growth medium. The minimal medium used was that described by Vogel (1956), except for those experiments in which the amount of sulphur in the medium was controlled. In such experiments, the sulphur free medium described in Strauss \& Minagawa (1959) was used and supplemented as indicated.

\section{RESULTS}

All our mutants with the exception of M3 were 'leaky'; that is, were able to synthesize some methionine even though they would not initiate growth on minimal medium even after 8 days incubation at $30^{\circ}$. The partial character of these mutants was demonstrated in two ways: first, by the ability of all the strains except M3 to continue growth when fed a limited amount of methionine (Fig. 2); and secondly, by the ability of all the mutants except M3 to incorporate ${ }^{35} \mathrm{SO}_{4}$ into methionine (Table 1). Incorporation of radioactive sulphate was demonstrated by the method of Strauss \& Minagawa (1959).

All of the mutant strains were able to utilize methionine as a sole source of sulphur with about the same efficiency as did the wild-type (Table 1). These strains therefore formed their cysteine from methionine as a sulphur source. Yet, all these strains required the addition of either cystathionine, homocysteine or methionine to minimal medium (containing sulphate) for rapid growth, indicating a nutritional block between cysteine and methionine. The rapid growth on methionine as a sole sulphur source therefore indicated that the sulphur of methionine could be converted to the sulphur of cysteine by a pathway which was not blocked. This has been formally represented as conversion to sulphate (Fig. 1), although there is no evidence that the sulphur is actually brought to this oxidation state.

Most strains were able to utilize S-methylcysteine as a source of sulphur amino acids as long as inorganic sulphate was absent from the medium. Strain M3 was the one strain unable to utilize this compound as a sulphur source (after about 10 days, even this strain produced some growth). S-methylcysteine was tested because of the report of Wiebers \& Garner (1960) that a methionine-requiring strain would grow with this supplement.

Very small amounts of sulphate inhibited the response of strains M7, 14 and 17 
(but not strain M4) to S-methylcysteine (Table 1, Fig. 3) and the sodium, potassium and ammonium sulphates inhibited equally well. Sulphite, thiosulphate and cysteine also inhibited the growth response to S-methylcysteine (Table 2).

\section{Genetic studies}

A reverted culture of $\mathbf{H 9 8}$ which grew on minimal medium was crossed to wildtype. On minimal medium a typical 6:2 ratio, characteristic of a suppressor

Table 1. Growth of methionine requiring mutants at $30^{\circ}$

\begin{tabular}{|c|c|c|c|c|c|c|c|c|c|}
\hline & & & & & & Growth & $\begin{array}{l}\text { ulphur } \\
\text { linagav }\end{array}$ & $\begin{array}{l}\text { medium } \\
\text { 1959) plus }\end{array}$ & (Strauss \& \\
\hline & $\begin{array}{l}\text { Three d } \\
\text { minimal }\end{array}$ & $\begin{array}{l}\text { s growth } \\
\text { edium (V } \\
\text { plus }\end{array}$ & $20 \mathrm{ml}$. & $\begin{array}{c}\text { Growth } \\
\text { on } \\
\text { limited }\end{array}$ & $\begin{array}{c}\text { Incorpora- } \\
\text { tion of } \\
{ }^{35} \mathrm{SO}_{4} \\
\text { into }\end{array}$ & $\begin{array}{c}\text { No } \\
\text { addition } \\
\text { (8 days } \\
\text { growth) }\end{array}$ & $\begin{array}{l}\text { Methio- } \\
\text { nine } \\
(1 \mathrm{mg} \cdot / \\
20 \mathrm{ml} .) \\
\text { (4. days) }\end{array}$ & $\begin{array}{c}\text { S-methyl- } \\
\text { cysteine } \\
(2 \mathrm{mg} . / \\
20 \mathrm{ml} .) \\
\text { (5 days) }\end{array}$ & $\begin{array}{c}\text { S methyl- } \\
\text { (ysteine } \\
(2 \mathrm{mg} . / \\
20 \text { ml.) }+ \\
\text { sulphate } \\
(100 \mu \mathrm{g} . \mathrm{S} / \\
20 \mathrm{ml} .) \\
\text { (5 days) }\end{array}$ \\
\hline & & Cysta- & Homo- & methio- & methio- & & ry wt. m & celium (mg. & \\
\hline 74.A & - & - & - & 62 & + & $2 \cdot 6$ & 73 & 50 & 125 \\
\hline M3 & 0 & + & + & 13 & 0 & 0 & 82 & o & 0 \\
\hline M4 & 0 & + & + & 47 & + & $0 \cdot 8$ & NT & 40 & 48 \\
\hline Mr & 0 & + & + & 77 & + & 0 & 52 & 12 & 0.2 \\
\hline M14 & $\mathbf{0}$ & 0 & + & 54 & + & 0 & 73 & 39 & 1 \\
\hline Mir & 0 & 0 & + & 73 & + & 0 & 62 & 53 & 22 \\
\hline H98 & 0 & 0 & + & NT & + & NT & NT & NT & NT \\
\hline
\end{tabular}

* Dry weight of mycelium per $20 \mathrm{ml}$. after 10 days in medium containing $0.2 \mathrm{mg}$. methionine/20 ml.

$\dagger$ The incorporation of ${ }^{35} \mathrm{SO}_{4}$ into methionine was determined as described in the legend to Fig. 4 and in Strauss \& Minagawa (1959).

$\mathbf{N T}=$ not tested.

Table 2. Effect of additional sulphur compounds on the response of mutant M14 to $S$-methylcysteine

Twenty ml. of sulphur free medium supplemented with $2 \mathrm{mg}$. of S-methylcysteine/ $20 \mathrm{ml}$. were added to all flasks. Incubation was in static culture at $30^{\circ}$.

\begin{tabular}{|c|c|c|c|}
\hline \multirow{3}{*}{$\begin{array}{l}\text { Additional compound } \\
\text { None }\end{array}$} & \multicolumn{3}{|c|}{$\begin{array}{cc}\text { M 14 } & \text { Wild-type 74 A } \\
\text { (7 days growth) } & (3 \text { days growth })\end{array}$} \\
\hline & $\begin{array}{l}\text { Amount added } \\
(\mu \mathrm{g} \mathrm{S})\end{array}$ & \multicolumn{2}{|c|}{ Dry wt. mycelium (mg.) } \\
\hline & - & 36 & 20 \\
\hline $\mathrm{Na}_{2} \mathrm{SO}_{4}$ & $\begin{array}{r}\mathbf{5 0} \\
\mathbf{5 0 0}\end{array}$ & $\begin{array}{l}2 \\
\cdot\end{array}$ & $\dot{72}$ \\
\hline$\left(\mathrm{NH}_{4}\right)_{2} \mathrm{SO}_{4}$ & $\begin{array}{r}50 \\
\mathbf{5 0 0}\end{array}$ & $\begin{array}{l}2 \\
\cdot\end{array}$ & 71 \\
\hline $\mathrm{Na}_{2} \mathrm{SO}_{3}$ & $\begin{array}{l}100 \\
500\end{array}$ & 18 & $\dot{73}$ \\
\hline $\mathrm{Na}_{2} \mathrm{~S}_{2} \mathrm{O}_{3}$ & $\begin{array}{r}\mathbf{5 0} \\
\mathbf{5 0 0}\end{array}$ & $\begin{array}{l}6 \\
\cdot\end{array}$ & $\dot{62}$ \\
\hline Cysteine & $\begin{array}{l}\mathbf{2 6 0} \\
\mathbf{2 6 0}\end{array}$ & $\begin{array}{l}0 \cdot 8 \\
\cdot\end{array}$ & 54 \\
\hline
\end{tabular}


mutation, was obtained (Table 3). The identity of the presumptive suppressor $\left(m e^{+} s u^{\text {198 }}\right)$ was verified by back crosses to H98. This suppressor mutant, when present with the $m e^{+}$allele, produced a partial deficiency for sulphur amino acids. It was stimulated by either cysteine or methionine as well as by cystathionine. This $m e^{+} s u^{\text {耳98 }}$ strain was inhibited by threonine which is itself evidence for a deficiency of sulphur amino acids in this strain, since threonine inhibition in Neurospora is counteracted by methionine (Doudney \& Wagner, 1952) and since wild-type is not threonine inhibited. In contrast to the behaviour of the $m e^{+} s u^{\text {H98 }}$ strain,

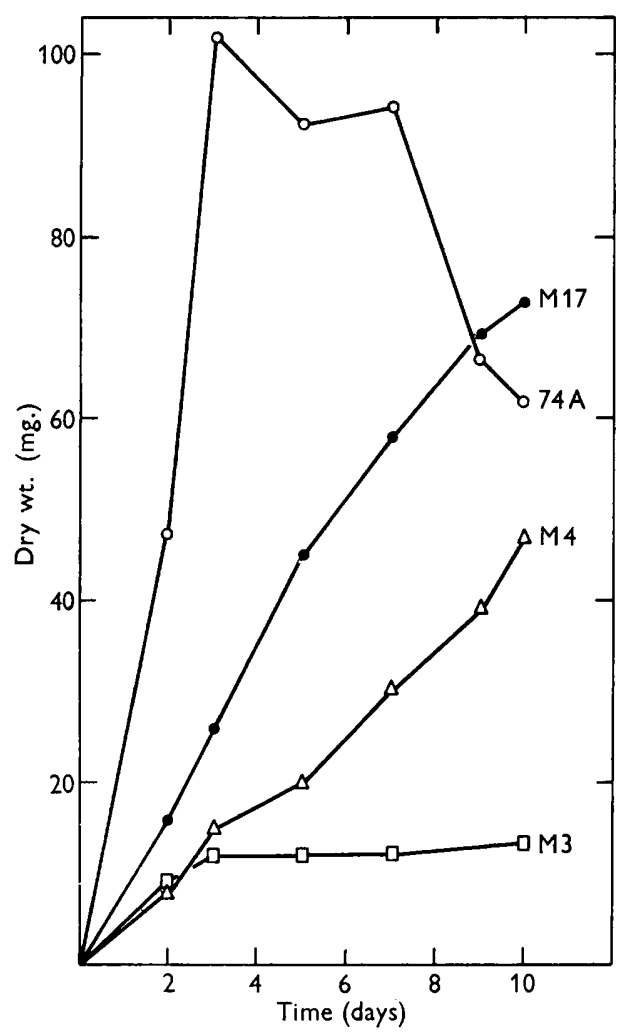

Fig. 2

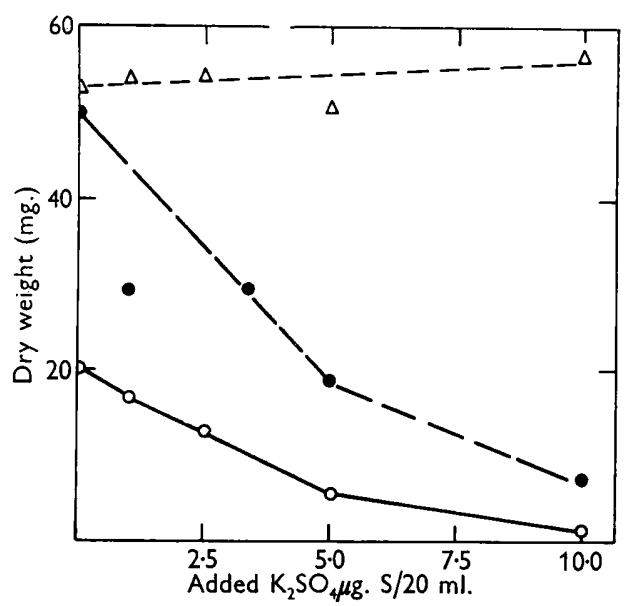

Fig. 3

Fig. 2. Growth response of a series of methionine-requiring mutants to limiting amounts of methionine. A series of flasks containing $0 \cdot 2 \mathrm{mg}$. L-methionine in $20 \mathrm{ml}$. minimal medium was inoculated and incubated in static culture at $30^{\circ}$. The top curve represents a wild-type control $(O)$ and the next curves represent the growth of M17 (०), M4 ( $\triangle$ ) and M3 ( $\square$ ) in descending order.

Fig. 3. The effect on the extent of growth of methionine-less mutant M14 of adding $\mathrm{K}_{2} \mathrm{SO}_{4}$ to sulphur-free medium containing S-methylcysteine. $\triangle$ represents growth of wildtype 74 $\mathrm{A}$ after 3 days at $30^{\circ}$ with $2 \mathrm{mg}$. S-methylcysteine $/ 20 \mathrm{ml}$. $\bullet$, O represent growth of M 14 after 5 days at $30^{\circ}$ with 4 and $1 \mathrm{mg}$. of S-methylcysteine/20 ml., respectively.

$m e-2^{\text {म98 }} s u^{\text {н98 }}$ was inhibited by both cysteine and cystathionine. We have been able to distinguish $m e^{+} s u$ strains from $m e s u$ strains by their different response to cysteine.

The suppressor isolated from H98 was crossed to strains M4 and M3 in order to determine whether the pattern of suppression reported by Giles (1951) represented 
a general finding. In confirmation of Giles' report (1951), we found that strain M 4 was suppressed by $s u^{\text {\#98 }}$ (Table $4 \mathrm{~A}$ ). The same pattern of stimulation by cysteine of the $m e^{+} s u$ strain and inhibition of the me $s u$ strain was apparent in this segregation. The cross $m e^{+} s u^{\mathrm{H} 98} \times m e^{\mathrm{M3}} s u^{+}$gave an ascus in which two of the four spore pairs were obvious wild-types, indicating that the other spore pairs were of the $m e^{\mathbf{M} 3} s u^{\text {म98 }}$ constitution. These cultures would not grow on minimal, indicating that they were not suppressed (but cf. below).

\section{Table 3. Segregation of the suppressor of $\mathrm{H} 98$}

Growth for 3 days at $30^{\circ}$ with $2 \mathrm{mg}$. supplement added/20 ml. minimal medium.

Cross :

\begin{tabular}{|c|c|c|c|c|c|c|c|}
\hline \multicolumn{8}{|c|}{ Cross: } \\
\hline & & \multicolumn{6}{|c|}{ Supplement } \\
\hline & & None & Cysteine & $\begin{array}{l}\text { Cysta- } \\
\text { thionine }\end{array}$ & $\begin{array}{l}\text { Homo- } \\
\text { cysteine }\end{array}$ & $\begin{array}{l}\text { Methio- } \\
\text { nine }\end{array}$ & Threonine \\
\hline Spore & Genetic & \multicolumn{6}{|c|}{ Dry wt. mycelium (mg.) } \\
\hline 1 & $m e^{+} s u^{+}$ & 92 & 97 & 98 & 61 & 87 & 109 \\
\hline 2 & $m e^{+} s u^{\text {H98 }}$ & $\mathbf{5 5}$ & 77 & 90 & 51 & 80 & 0 \\
\hline 3 & $m e-2^{\mathrm{H} 98} s u^{\mathrm{H} 98}$ & 21 & 9 & 0.9 & 18 & 70 & 0 \\
\hline 4 & $m e-2^{\text {H98 }} \mathrm{su}^{+}$ & 0 & $\mathbf{0}$ & $\mathbf{0}$ & 5 & 40 & 0 \\
\hline
\end{tabular}

Table 4. Suppression of methionine requiring mutants by suppressors from non-homologous strains

Recorded dry weights produced after 3 days at $30^{\circ}$ in 20 ml. of minimal medium with $2 \mathrm{mg}$. of the supplement shown.

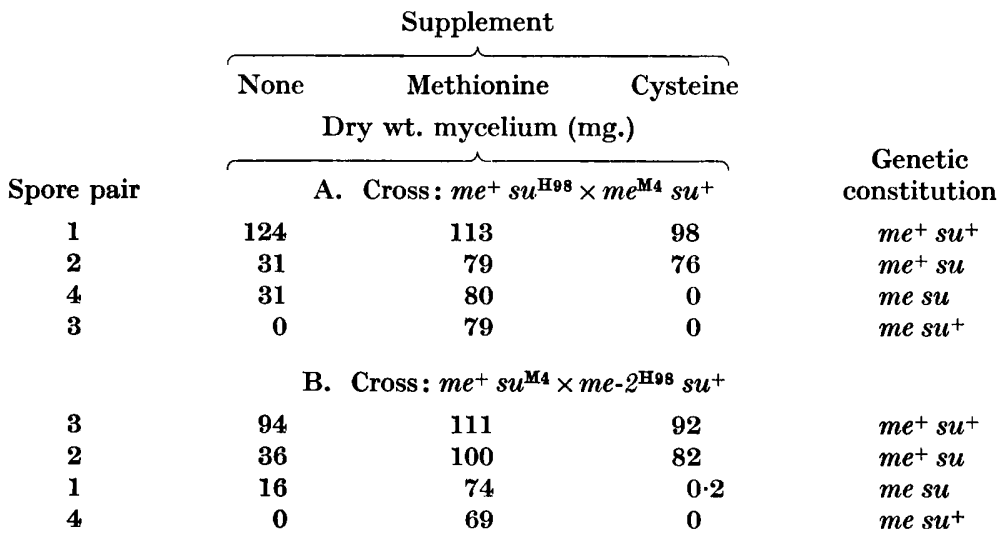

We were also interested in determining whether a suppressor isolated from $\mathbf{M}_{4}$ (blocked between cysteine and cystathionine) would suppress strain H98 (blocked between cystathionine and homocysteine). Such a suppressor was isolated by the method of Yanofsky \& Bonner (1955) and its identity was established by appropriate segregation tests. This $m e^{+} s u^{\mathbf{M} 4}$ strain was also stimulated by sulphur amino acids and inhibited by threonine in a manner similar to the $s u^{\text {म98 }}$ strain (Table 5). The $m e^{\mathrm{M} 4} s u^{\mathrm{M} 4}$ strain was inhibited by cysteine but stimulated by cystathionine. 
The suppressor isolated from M4 suppressed H98 (Table 4B) just as the suppressor isolated from H98 suppressed M4, but these two suppressors were not alleles. The cross $m e^{+} s u^{\mathrm{H} 98} \times m e^{+} s u^{\mathrm{M4}}$ gave 25 wild-type cultures not inhibited by threonine out of the 96 single ascospore cultures isolated, indicating that these two factors were not alleles, notwithstanding their similar phenotypic effects.

The $m e^{\mathbf{M}_{3}} s u^{\mathbf{M} 4}$ strain was not suppressed, which also indicated the similarity in action of the $s u^{\mathbf{H} 98}$ and $s u^{\mathbf{M 4}}$ genes. However, M3 strains carrying either $s u$ gene were different from the $m e^{\mathrm{M} 3} s u^{+}$strain since they did grow with S-methylcysteine as a supplement, in contrast to M3 strains not carrying a suppressor gene.

Table 5. Response of strains carrying su $\mathrm{su}^{\mathrm{M} 4}$ to amino acids

Growth after 3 days at $30^{\circ}$ on minimal medium.

\begin{tabular}{|c|c|c|c|c|c|c|}
\hline \multirow{3}{*}{$\begin{array}{c}\text { Genetic } \\
\text { constitution }\end{array}$} & \multicolumn{6}{|c|}{ Supplement (2 mg./20 ml.) } \\
\hline & None & Cysteine & $\begin{array}{l}\text { Cysta- } \\
\text { thionine }\end{array}$ & $\begin{array}{l}\text { Homo- } \\
\text { cysteine }\end{array}$ & $\begin{array}{l}\text { Methio- } \\
\text { nine }\end{array}$ & Threonine \\
\hline & \multicolumn{6}{|c|}{ Dry wt. mycelium (mg.)/20 ml. medium } \\
\hline$m e^{+} s u^{M_{4}}$ & 62 & 108 & 92 & 104 & 112 & $7 \cdot 2$ \\
\hline$m e^{\mathrm{M} 4} s u^{\mathrm{M} 4}$ & 20 & $\mathbf{0}$ & 59 & 60 & 92 & $\mathbf{0}$ \\
\hline
\end{tabular}

Table 6. Stimulation by S-methylcysteine of suppressed mutants inhibited by cysteine

Growth for 3 days at $30^{\circ}$ in minimal medium containing sulphate (Vogel, 1956).

\begin{tabular}{|c|c|c|c|c|c|}
\hline \multirow[b]{3}{*}{ Strain } & \multicolumn{5}{|c|}{ Supplement per $20 \mathrm{ml}$. medium } \\
\hline & \multirow{3}{*}{ None } & \multicolumn{2}{|c|}{ Cysteine } & \multicolumn{2}{|c|}{$S$-Methylcysteine } \\
\hline & & $0.5 \mathrm{mg}$. & $2.0 \mathrm{mg}$. & $0.5 \mathrm{mg}$. & $2.0 \mathrm{mg}$. \\
\hline & & \multicolumn{4}{|c|}{ Dry wt. mycelium (mg.)/20 ml. medium } \\
\hline$m e^{\mathrm{M} 4} s u^{\mathrm{M} 4}$ & 18 & $\mathbf{0}$ & $\mathbf{0}$ & 18 & 81 \\
\hline$m e^{\mathrm{M}_{4}} s u^{\mathrm{H} 98}$ & 8 & 0 & $\mathbf{0}$ & 21 & 25 \\
\hline
\end{tabular}

\section{Studies with $S$-methylcysteine}

Methionine will serve as a source of sulphur for all the methionine-requiring mutants tested. It seemed likely that S-methylcysteine served as a source of methionine without passing through cysteine and that the methionine formed could then act as a source of cysteine for growth.

We have been able to show that S-methylcysteine added to medium containing radioactive sulphate and limiting amounts of methionine lowered the incorporation of ${ }^{35} \mathrm{SO}_{4}$ into methionine by methionine-requiring mutants more than into cysteine (Fig. 4). The ratio of total counts in the areas methionine + methionine sulphoxide to cysteine + cysteic acid was determined after elution and gave the following values: H98, 2.32; H98+S-methylcysteine, 1.20; M4, 2.14; M4+S-methylcysteine, 1·03. These results indicate that the sulphur of methylcysteine is incorporated more efficiently into methionine than into cysteine. Roberts et al. (1955) reported that $S$-methylcysteine could lower the uptake of radioactive sulphate to 
$17 \%$ of the control and that it lowered the uptake of ${ }^{35} \mathrm{~S}$ cystine to $71 \%$ of the control.

S-methylcysteine stimulates the growth of $m e^{\mathrm{M} 4} s u$ strains which show inhibition by cysteine (Table 6 ). In addition, and as described above (Table 2 ), cysteine inhibits the response of M14 to S-methylcysteine.

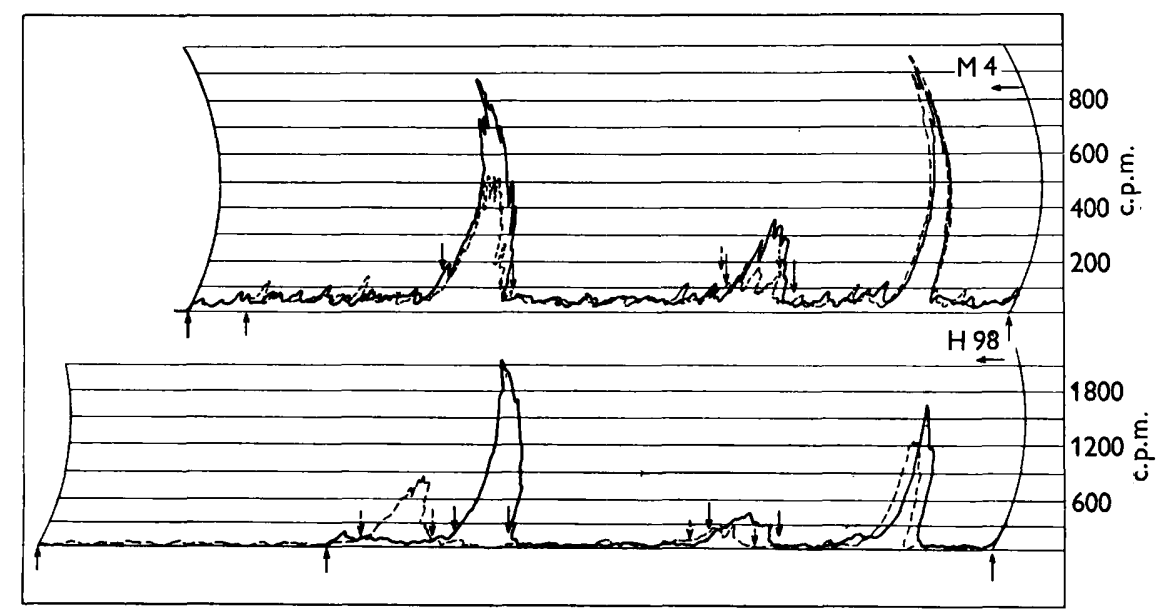

Fig. 4. Effect of S-methylcysteine on the incorporation of ${ }^{25} \mathrm{SO}_{4}$ into sulphur amino acids by leaky methionine-requiring mutants of Neurospora. Strains H98 and M4 were grown for 5 days on $20 \mathrm{ml}$. of a sulphur-free medium supplemented with $500 \mu \mathrm{g}$. of inorganic sulphur (as $\mathrm{K}_{2} \mathrm{SO}_{4}$ ) per $20 \mathrm{ml}$. and containing $0.2 \mathrm{mg}$. of L-methionine and $15 \mu \mathrm{c}$. of ${ }^{35} \mathrm{SO}_{4}$. S-methyl-L-cysteine was added at a concentration of $2 \mathrm{mg} . / 20 \mathrm{ml}$. where indicated. The mycelium was harvested, washed with hot $50 \%(v / v)$ ethanol, then with hot water, $0.5 \mathrm{M}-\mathrm{HClO}_{4}\left(7^{\circ}\right)$, ethanol and ether. After drying, the mycelium was autoclaved at 15 PS1 with $6 \mathrm{~N}-\mathrm{HCl}$ for $2 \mathrm{hr}$. in a sealed glass tube. Excess HCl was removed by repeated evaporation in vacuo and the hydrolysate was taken up in water and chromatographed by the descending technique on Whatman no. 1 paper with butanol + acetic acid + water $(2+1+1)$. Typical $R_{F}$ values with this solvent are : cysteine, 0.2 ; cysteic acid, 0.2 ; methionine sulphoxide, 0.42 ; S-methylcysteine, 0.57 ; and methionine, 0.68-0.7. Both methionine and methionine sulphoxide were chromatographed along with the hydrolysates and their position is indicated by arrows on the tracings as are the boundaries of the chromatograms. The position of the radioactive spots was located and traced as previously described (Strauss \& Minagawa, 1959). Solid line = material grown with no S-methylcysteine. Dotted line = material grown with Smethylcysteine. The horizontal arrows indicate the direction of solvent flow and of the scanning. The vertical arrows pointing upward indicate the origin and final solvent fronts respectively. The vertical arrows pointing downwards indicate the positions of non-radioactive methionine sulphoxide (lower $R_{F}$ ) and methionine (higher $R_{F}$ ) cochromatographed with the radioactive material. Solid arrows show the position of the compounds co-chromatographed with material grown in the absence of S-methylcysteine, dotted arrows indicate the location of compounds co-chromatographed with material grown in the presence of S-methylcysteine.

\section{DISCUSSION}

The response of suppressed strains to the sulphur amino acids is summarized in Table 7. Two strains were studied in detail, strain M4 blocked between cysteine and cystathionine, and strain $\mathbf{H 9 8}$ blocked between cystathionine and homocysteine. In addition, two non-allelic suppressors were isolated, $s u^{\mathbf{M 4}}$ and $s u^{\mathbf{H 9 8}}$. Both suppressors suppress both mutants M 4 and H98 and neither suppressor will 
suppress M3, a non-leaky mutant. Both $m e^{+} s u$ strains grew less well on minimal medium than the unsuppressed wild-type but were stimulated in their growth by the sulphur amino acids. The me su mutants, on the other hand, were inhibited by certain of the sulphur amino acids which in the case of both me mutants were restricted to those amino acids located in the metabolic scheme before the point of the original nutritional block. Sulphur amino acids located after the point of the block either stimulated or had no noticeable effect. The same pattern of inhibition was obtained regardless of the origin of the suppressor. All the strains listed in Table 7 were inhibited by threonine.

\section{Table 7. Response of suppressed strains to additions to the medium}

Both $m e^{+} s u$ strains were stimulated by the compounds indicated. All strains were stimulated by homocysteine or methionine.

\begin{tabular}{|c|c|c|}
\hline \multirow[b]{2}{*}{ Genetic constitution } & \multicolumn{2}{|c|}{ Addition } \\
\hline & Cysteine & Cystathionine \\
\hline$m e-2^{\mathrm{H98}} s u^{+}$ & - & - \\
\hline$m e^{\mathrm{M} 4} s u^{+}$ & 一 & $\mathbf{S}$ \\
\hline$m e-2^{\mathrm{H} 98} s u^{\mathrm{H} 98}$ & $\mathbf{I}$ & $\mathbf{I}$ \\
\hline$m e^{\mathrm{M} 4} s u^{\mathrm{H} 98}$ & $\overline{\mathbf{I}}$ & $\mathbf{S}$ \\
\hline$m e-2^{\mathrm{H} 98} s u^{\mathrm{M} 4}$ & I & $\mathbf{I}$ \\
\hline$m e^{\mathrm{M} 4} s u^{\mathrm{M} 4}$ & I & $\mathbf{S}$ \\
\hline
\end{tabular}

The fact that $m e^{+} s u$ strains are stimulated by sulphur amino acids indicates that they are partially deficient in their ability to make these substances. The methionine mutants which can be suppressed are themselves leaky. A possible explanation of the suppression is that, as a result of the me mutation, an inhibitor related to cysteine accumulates which reinforces the block to methionine synthesis. The combination of the leaky me mutation with the $s u$ mutations lowers the amount of inhibitor which can be formed since, as demonstrated nutritionally, the $s u$ strains are unable to make cysteine and other sulphur amino acids at a normal rate since these compounds stimulate growth. The hypothesis accounts for the behaviour of the me su strains when confronted with potential inhibitors. Compounds coming after the point of the block stimulate, since they can be readily converted to methionine; compounds coming before the point of the block inhibit, since they can be converted to the inhibitor even in the presence of the $s u$ gene. According to this hypothesis, only leaky mutants should be suppressible by these suppressors, but it is not necessary that all leaky mutants be suppressed. Non-leaky mutants appear to be relatively rare, although they can be obtained (no $m e-2$ mutant which is not leaky has been obtained by us or is available from the Neurospora Stock Centre at Dartmouth).

We suppose that a block between cystathionine and homocysteine or between cysteine and cystathionine brings about equivalent internal inhibition and that the production of this state of internal inhibition is prevented by either $s u$ gene. Such an action by the $s u$ gene might account for the restoration of cystathionase I and II activities observed in me mutants by Fischer (1957). This scheme is analogous to that devised to account for the behaviour of the suppressors of the acetate-requiring 
mutants of Neurospora (Strauss \& Pierog, 1954). It was supposed at that time that the acetate suppressors lowered the production of an inhibitor (acetaldehyde) formed in excess as a result of the first mutation. We suppose that genetic suppression by the interaction of the cytoplasmic reactions of intermediary metabolism is not a particularly uncommon mechanism. However, as Yanofsky (1961) has pointed out, selection of a suppressor on minimal medium eliminates any genes which suppress one mutation at the cost of introducing a new requirement. It is not possible to make any statements about the quantitative prevalence of particular types of gene interaction as a result of the compilation of cases of suppressors picked by a particular selective method.

The data presented in this paper make it very likely that the sulphur of S-methylcysteine can be converted to methionine without passing through cysteine. Cysteine inhibits me su double mutants, S-methylcysteine stimulates them. A number of methionine-requiring mutants respond to $S$-methylcysteine as a sole nutritional supplement; both sulphate and cysteine inhibit this response. S-methylcysteine lowers the incorporation of ${ }^{{ }^{5} \mathrm{SO}_{4}}$ into methionine proportionally more than cysteine. These data are most easily explained by supposing that S-methylcysteine may be more or less directly converted into methionine without passing through cysteine. A possible scheme for this conversion has been suggested by Wiebers \& Garner (1960).

Some time ago we supposed (Strauss \& Minagawa, 1959) that the methionine synthesized by a leaky methionine-requiring mutant was formed via the pathway cysteine-cystathione-homocysteine-methionine. We based our conclusion upon the accumulation and then gradual disappearance of cystathionine when our strain (584) was grown with limiting amounts of sulphur. However, S-methylcysteine has been reported as a natural metabolite in Neurospora (Ragland \& Liverman, 1956) and it is now apparent that the methionine formed by leaky mutants could come via an alternate pathway based on this compound. In fact, the existence of two pathways to methionine could help to account for the surprisingly high proportion of leaky methionine-requiring mutants. An alternate pathway based on S-methylcysteine might be inhibited or repressed in the ordinary course of events and the suppressor genes might act by removing a feedback inhibitor or repressor, thereby permitting the alternate pathway to function. Those mutants which are non-leaky might then be supposed to be blocked before the point at which the alternate pathway diverges from the standard, although it is possible that mutants exist, blocked at this position, which are leaky for other reasons. The two absolute mutants now available are blocked between cysteine and cystathionine. It has always been puzzling to note that three non-allelic genes are located at what has been presumed to be a single metabolic step reaction (Barrat et al. 1954); the proposed alternate pathway is presumably not operative in all three. Our conclusion is, therefore, similar to that of Wiebers \& Garner (1960) and of Maw (1961).

Although we find the sulphate inhibition of S-methylcysteine utilization to be an interesting phenomenon and have used it in our argument above, its metabolic basis is at present completely unknown. Some strains are resistant to sulphate inhibition while others, blocked in what is apparently the same position, are susceptible. It is likely that an explanation must wait for an enzymic description of these phenomena. 
Since submitting this paper we have completed additional genetic tests on the suppression of known me mutants obtained from the Neurospora stock centre. Mutants blocked at the $m e-2, m e-3$ and $m e-7$ loci were suppressed by both $s u^{\text {H98 }}$ and $s u^{\mathrm{M4}}$. Two mutants at the $m e-5$ locus, one leaky and one non-leaky, were not suppressed. We do not yet have sufficient data to assert that our $m e^{\mathrm{M} 3}$ is an $m e-5$ allele.

Dr Noreen Murray (personal communication) has tested the majority of her $m e-2$ mutants and finds them all leaky, only one of six me-5 mutants was leaky, whereas the $m e-3$ and $m e-7$ strains tested were leaky.

This work was supported by funds provided by the National Science Foundation and by a grant from the Dr Wallace C. and Clara A. Abbott Memorial Fund of The University of Chicago.

\section{REFERENCES}

Barratt, R. W., Newmeyer, D., Perkins, D. \& Garnjobst, L. (1954). Map construction in Neurospora crassa. Advanc. Genet. 6, 1.

Doudney, C. \& Wagner, R. (1952). Threonine inhibition in a strain of Neurospora. Proc. nat. Acad. Sci., Wash. 38, 196.

Fischer, G. A. (1957). The cleavage and synthesis of cystathionine in wild-type and mutant strains of Neurospora crassa. Biochim. biophys. Acta, 25, 50.

Giles, N. (1951). Studies on the mechanism of reversion in biochemical mutants of Neurospora crassa. Cold Spr. Harb. Symp. quant. Biol. 16, 283.

Maw, G. A. (1961). Ability of $S$-methyl-L-cysteine to annul the inhibition of yeast growth by L-ethionine and by $S$-ethyl-L-cysteine. J. gen. Microbiol. 25, 441.

RAGLAND, J. B. \& Liverman, J. L. (1956). S-Methyl-L-cysteine as a naturally occurring metabolite in Neurospora crassa. Arch. Biochem. Biophys. 65, 574.

Roberts, R. B., Abelson, P. H., Cowie, D. B., Bolton, E. B. \& Britten, R. J. (1955). Studies of biosynthesis in Escherichia coli. Carneg. Inst. Publ. no. 607, p. 400.

Strauss, B. (1955). A mechanism of gene interaction. Amer. Nat. 89, 141.

Strauss, B. \& Minagawa, T. (1959). The formation of methionine by a methionine-requiring mutant of Neurospora crassa. J. gen. Microbiol. 20, 237.

Strauss, B. \& Pierog, S. (1954). Gene interactions: the mode of action of the suppressor of acetate-requiring mutants of Neurospora crassa. J. gen. Microbiol. 10, 221.

VogeL, H. J. (1956). A convenient growth medium for Neurospora. Microbial Genetics Bull. 13, 42.

Wiebers, J. L. \& Garner, H. R. (1960). Metabolic relationship between cystathionine and methionine in Neurospora. J. Bact. 80, 50.

Woodward, V. W., DeZeeuw, J. R. \& SRb, A. M. (1954). The separation and isolation of particular biochemical mutants of Neurospora by differential germination of conidia followed by filtration and selective plating. Proc. nat. Acad. Sci., Wash. 40, 192.

YANOFSKY, C. (1961). The effects of mutation on the composition and properties of the A protein of Escherichia coli tryptophan synthetase. Cold Spr. Harb. Symp. quant. Biol. 26 (in the Press).

Yanofsky, C. \& BonNer, D. M. (1955). Gene interaction in tryptophan synthetase formation. Genetics, $\mathbf{4 0 ,} 761$. 